\title{
ON THE IMAGINARY ROOTS OF A POLYNOMIAL AND THE REAL ROOTS OF ITS DERIVATIVE*
}

\author{
BY \\ HENRY BEDINGER MITCHELL
}

1. Introduction. If a polynomial with real coefficients is found to have bend points exterior to the interval within which its real roots must lie, or if its derivative vanishes more than once in an interval between two consective real roots, the existence of imaginary roots is immediately evident. It would seem probable that such marked features of the graph, as thus instantly reveal the presence of imaginary roots, should also set limits, in the complex plane, to a region within which one or more of these roots must be contained; and the question arises as to what we can infer, without laborious calculation, as to their position. In the theorems which follow, we shall seek a partial answer to this question, and shall therefore be concerned, not with the problem of obtaining the closest possible approximations, but rather with the inferences that can be drawn from certain minima data, that are to include the existence of a bend point, where the curve is not concave toward the $X$-axis, and its position relative to the adjacent real root or roots.

2. Notation. Let

$$
f(x) \equiv A_{0} x^{n}+A_{1} x^{n-1}+\cdots+A_{n-1} x+A_{n}
$$

be a polynomial having real coefficients, and let

$$
\begin{array}{r}
\phi(x) \equiv\left(x-r_{1}\right)\left(x-r_{2}\right) \cdots\left(x-r_{m}\right) \quad\left(r_{1} \leqq r_{2} \leqq \cdots \leqq r_{m}\right), \\
\psi(x) \equiv\left[\left(x-a_{1}\right)^{2}+b_{1}^{2}\right] \cdots\left[\left(x-a_{k}\right)^{2}+b_{k}^{2}\right] \quad(2 k=n-m)
\end{array}
$$

be the products of the factors corresponding, respectively, to its real and imaginary roots, so that

$$
f(x) \equiv A_{0} \phi(x) \cdot \psi(x) .
$$

We shall then denote by $L(x)$ and $\lambda(x)$, respectively, the logarithmic derivatives of $\phi(x)$ and $\psi(x)$, so that

$$
\frac{f^{\prime}(x)}{f(x)} \equiv L(x)+\lambda(x)
$$

* Presented to the Society, October 28, 1916. 
where

$$
\begin{gathered}
L(x) \equiv \sum_{i=1}^{m} \frac{1}{x-r_{i}}, \\
\lambda(x) \equiv \sum_{i=1}^{k} \frac{2\left(x-a_{i}\right)}{\left(x-a_{i}\right)^{2}+b_{i}^{2}} .
\end{gathered}
$$

3. Theorem I. If $f(x)$ be a polynomial having real coefficients and at least one real root, and if $f^{\prime}$ have a real root $\rho$ exterior to the interval $r_{1} \leqq x \leqq r_{m}$, between the least and the greatest real root of $f$, then there exists at least one pair of imaginary roots of $f, a \pm i b$, whose real and imaginary parts satisfy the inequalities

or

$$
\begin{gathered}
0<a-\rho<2 h\left(\rho-r_{m}\right), \\
0<b \leqq h\left(\rho-r_{m}\right)
\end{gathered}
$$

(when $\rho>r_{m}$ ),

$$
\begin{gathered}
0<\rho-a<2 h\left(r_{1}-\rho\right), \\
0<b \leqq h\left(r_{1}-\rho\right)
\end{gathered}
$$

(when $\left.\rho<r_{1}\right)$,

where $2 h$ is the number of imaginary roots whose real part is greater than $\rho$ in the first case, and less than $\rho$ in the second case.

That $f$ has imaginary roots is an immediate consequence of Rolle's Theorem. As $\rho$ is a root of $f^{\prime}$ that is not a root of $f$, we may write, from (4),

$$
L(\rho)+\lambda(\rho)=0 .
$$

When $\rho>r_{m}$ this gives

$$
0<\frac{1}{\rho-r_{m}} \leqq \sum_{i=1}^{m} \frac{1}{\rho-r_{i}}=2 \sum_{i=1}^{k} \frac{a_{i}-\rho}{\left(a_{i}-\rho\right)^{2}+b_{i}^{2}} .
$$

The separate terms of the last sum are positive or negative according as $a_{i} \gtrless \rho$, and as the whole sum is positive at least one of its terms must be so also. Denoting by $h \leqq k$ the number of such positive terms, and by $a \pm i b$ the pair of imaginary roots corresponding to a term that is at least as great as any other, we have

whence

$$
0<\frac{1}{\rho-r_{m}} \leqq 2 h \frac{a-\rho}{(a-\rho)^{2}+b^{2}}<\frac{2 h}{a-\rho},
$$

$$
0<a-\rho<2 h\left(\rho-r_{m}\right) \text {. }
$$

To obtain the inequality for $b$ we have only to note that

$$
\frac{2(a-x)}{(a-x)^{2}+b^{2}}
$$


has the maximum value of $1 / b$, so that

and therefore, by (9),

$$
2 h \frac{a-\rho}{(a-\rho)^{2}+b^{2}} \leqq \frac{h}{b},
$$

$$
0<b \leqq h\left(\rho-r_{m}\right),
$$

which completes the proof for the case where $\rho>r_{m}$, and a precisely similar procedure establishes the inequalities (8) for $\rho<r_{1}$.

CoRollary. As $1 \leqq h \leqq k$, where $2 k$ is the number of imaginary roots of $f$, we may substitute $k$ for $h$ in. (7) and (8), which gives the form in which the theorem must be used when we have no indication of how the imaginary roots of $f$ are separated.

4. Theorem II. If $f(x)$ be a polynomial having real coefficients and at least one real root, and if $f^{\prime}$ have a real root $\rho$ exterior to the interval $r_{1} \leqq x \leqq r_{m}$ between the least and the greatest real root of $f$, then $f^{\prime}$ has an even number of such roots, without and on the same side of the interval, and there exists at least one pair of imaginary roots of $f, \alpha \pm i \beta$, whose real and imaginary parts satisfy the inequalities

$$
\begin{array}{r}
\left|\rho_{2}-\alpha\right|<\beta \leqq\left(\rho_{2}-r_{m}\right) \sqrt{2 k}, \\
\left|\rho_{2}-\alpha\right| \leqq\left(\rho_{2}-r_{m}\right) \frac{\sqrt{k}}{2},
\end{array}
$$

where $\rho_{2}>r_{m}$ is the greatest real root of $f^{\prime}$, or

$$
\begin{array}{r}
\left|\rho_{2}-\alpha\right|<\beta \leqq\left(r_{1}-\rho_{2}\right) \sqrt{2 k}, \\
\left|\rho_{2}-\alpha\right| \leqq\left(r_{1}-\rho_{2}\right) \frac{\sqrt{k}}{2},
\end{array}
$$

where $\rho_{2}<r_{1}$ is the least real root of $f^{\prime}$, and $2 k$ is the number of imaginary roots of $f$.

It will be sufficient, as before, to establish the first set of inequalities, the proof for the second case being similar save for the change of sign. From (4) we have

$$
\frac{f^{\prime}}{f} \equiv L+\lambda \equiv \sum_{i=1}^{m} \frac{1}{x-r_{i}}+\sum_{i=1}^{k} \frac{2\left(x-a_{i}\right)}{\left(x-a_{i}\right)^{2}+b_{i}^{2}} .
$$

For values of $x$ greater than both $r_{m}$ and the greatest $a$ in $\lambda$, both $L$ and $\lambda$ are positive. For sufficiently small values of the positive quantity $\delta, L+\lambda$ is also positive when $x=r_{m}+\delta$ and is finite and continuous for all greater values of $x$. Therefore if $f^{\prime}$, and so $L+\lambda$, have one real root greater than $r_{m}$ it must have an even number of such roots, and at the greatest of these the slope of $L+\lambda$ cannot be negative. 
We have, therefore,

whence

$$
L^{\prime}\left(\rho_{2}\right)+\lambda^{\prime}\left(\rho_{2}\right) \geqq 0,
$$

$$
0<\frac{1}{\left(\rho_{2}-r_{m}\right)^{2}} \leqq \sum_{i=1}^{m} \frac{1}{\left(\rho_{2}-r_{i}\right)^{2}} \leqq 2 \sum_{i=1}^{k} \frac{b_{i}^{2}-\left(\rho_{2}-a_{i}\right)^{2}}{\left[b_{i}^{2}+\left(\rho_{2}-a_{i}\right)^{2}\right]^{2}} .
$$

As the last sum is positive, at least one of its terms must be greater than zero. Denoting by $\alpha \pm i \beta$ the pair of imaginary roots corresponding to a positive term that is at least as great as any other, we may write

and

$$
\beta^{2}>\left(\rho_{2}-\alpha\right)^{2},
$$

$$
0<\frac{1}{\left(\rho_{2}-r_{m}\right)^{2}} \leqq 2 k \frac{\beta^{2}-\left(\rho_{2}-\alpha\right)^{2}}{\left[\beta^{2}+\left(\rho_{2}-\alpha\right)^{2}\right]^{2}}
$$

If we regard the last expression as a function of $\beta$, its maximum value is found to be

$$
\frac{k}{4\left(\rho_{2}-\alpha\right)^{2}}
$$

and we observe that it is also less than, or at most equal to, $2 k / \beta^{2}$, from which we obtain

whence

$$
\begin{gathered}
0<\frac{1}{\left(\rho_{2}-r_{m}\right)^{2}} \leqq \frac{k}{4\left(\rho_{2}-\alpha\right)^{2}}, \\
\frac{1}{\left(\rho_{2}-r_{m}\right)^{2}} \leqq \frac{2 k}{\beta^{2}},
\end{gathered}
$$

$$
\begin{gathered}
\left|\rho_{2}-\alpha\right| \leqq\left(\rho_{2}-r_{m}\right) \frac{\sqrt{k}}{2}, \\
\left|\rho_{2}-\alpha\right|<\beta \leqq\left(\rho_{2}-r_{m}\right) \sqrt{2 k} .
\end{gathered}
$$

5. Theorem III. If a polynomial, $f(x)$, having real coefficients, have at least one real root and not more than two imaginary roots, and if $f^{\prime}$ have a real root $\rho$ greater than the greatest real root of $f$ or less than its least real root, then $f^{\prime}$ has two and only two such roots, $\rho_{1}$ and $\rho_{2}$, both being greater than the greatest real root of $f$ or both less than its least real root, and $f$ has a pair of imaginary roots, $a \pm i b$, such that

$$
\begin{array}{lll}
0<a-\rho_{2}<b \leqq \rho_{1}-r_{m} & \text { if } & r_{m}<\rho_{1} \leqq \rho_{2}, \\
0<\rho_{2}-a<b \leqq r_{1}-\rho_{1} & \text { if } & \rho_{2} \leqq \rho_{1}<r_{1},
\end{array}
$$

so that, of the projections on the $X$-axis of the two exterior bend points, the one lies nearer to the real part of the imaginary roots of $f$ than the other does to the nearest real root of $f$. 
It will be sufficient to observe that as $f$ has but a single pair of imaginary roots, the inequalities of Theorems I and II must alike apply to that pair for $h=k=1$.

Corollary. By Theorem II, we may also write for $k=1$,

$$
\begin{aligned}
\rho_{2}<a \leqq \frac{3 \rho_{2}-r_{m}}{2} & \text { when } & r_{m}<\rho_{1} \leqq \rho_{2}, \\
\rho_{2}>a \geqq \frac{3 \rho_{2}-r_{1}}{2} & \text { when } & \rho_{2} \leqq \rho_{1}<r_{1},
\end{aligned}
$$

so that, when $f$ has but a single pair of imaginary roots, if the interval on the $X$-axis from the most remote exterior real root of $f^{\prime}$ to the nearest real root of $f$ be divided externally in the ratio of $-1: 3$, the real part of the imaginary roots of $f$ will lie between the root of $f^{\prime}$ and the point of division.

6. Theorem IV. If $f(x)$ be a polynomial having real coefficients and not more than eight imaginary roots, and if $f^{\prime}$ reduce to zero more than once in an interval between two distinct, consecutive, real roots of $f, r_{h}<x<r_{i}$, then there exists a pair of imaginary roots of $f, \alpha \pm i \beta$, such that $\alpha$ is included in the interval, and $\beta$ is not greater than its extent:

$$
r_{h}<\alpha<r_{i}, \quad \text { and } \quad 0<\beta \leqq r_{i}-r_{h} .
$$

As $f^{\prime}$, and so $L+\lambda$, reduces to zero more than once in the interval, there must be an intermediate point $x=c$, for which $L^{\prime}(c)+\lambda^{\prime}(c)=0$, or

But

$$
\sum_{i=1}^{m} \frac{1}{\left(c-r_{i}\right)^{2}}=2 \sum_{i=1}^{k} \frac{b_{i}^{2}-\left(c-a_{i}\right)^{2}}{\left[b_{i}^{2}+\left(c-a_{i}\right)^{2}\right]^{2}} \text {. }
$$

$$
\sum_{i=1}^{m} \frac{1}{\left(c-r_{i}\right)^{2}} \geqq \frac{1}{\left(c-r_{h}\right)^{2}}+\frac{1}{\left(c-r_{i}\right)^{2}} \geqq \frac{8}{\left(r_{i}-r_{h}\right)^{2}} .
$$

As the first member of (17) is positive, one or more of the terms of the second member must be positive also. Denoting by $\alpha \pm i \beta$ the pair of imaginary roots of $f$ corresponding to a positive term that, is at least as great as any other, we have, as in Theorem II, remembering that $2 k \leqq 8$,

$$
\begin{gathered}
2 \sum_{i=1}^{k} \frac{b_{i}^{2}-\left(c-a_{i}\right)^{2}}{\left[b_{i}^{2}+\left(c-a_{i}\right)^{2}\right]^{2}} \leqq \\
2 k \frac{\beta^{2}-(c-\alpha)^{2}}{\left[\beta^{2}+(c-\alpha)^{2}\right]^{2}} \leqq \frac{k}{4(c-\alpha)^{2}} \leqq \frac{1}{(c-\alpha)^{2}} \\
2 k \frac{\beta^{2}-(c-\alpha)^{2}}{\left[\beta^{2}+(c-\alpha)^{2}\right]^{2}} \leqq \frac{2 k}{\beta^{2}} \leqq \frac{8}{\beta^{2}}
\end{gathered}
$$

Combining (19) with (17) and (18), we have

$$
\beta \leqq r_{i}-r_{h}, \quad \frac{1}{(c-\alpha)^{2}} \geqq \frac{1}{\left(c-r_{h}\right)^{2}}+\frac{1}{\left(c-r_{i}\right)^{2}} .
$$


Thus $1 /(c-\alpha)^{2}$ is greater than both $1 /\left(c-r_{h}\right)^{2}$ and $1 /\left(c-r_{i}\right)^{2}$, and hence $|c-\alpha|$ is less than $\left|c-r_{h}\right|$ and less, also, than $\left|c-r_{i}\right|$. As $c$ lies within the interval from $r_{h}$ to $r_{i}$, so also does $\alpha$.

Corollary. When $f(x)$ has but two imaginary roots, then

$$
\beta \leqq \frac{r_{i}-r_{h}}{2}
$$

7. Theorem V. If $f(x)$ be a polynomial having real coefficients, and if $f^{\prime}$ vanish more than once in an interval $r_{h}<x<r_{i}$ between two distinct, consecutive real roots of $f$, the real parts of the imaginary roots of $f$ cannot all lie without and on the same side of the interval, unless the number of real roots on the opposite side be greater than eight,* and unless the number of imaginary roots be also greater than eight, the degree of the polynomial being thus at least twenty.

The necessary and sufficient condition that $f^{\prime}$ should vanish more than once in the interval is that there should be a point $c, r_{h}<c<r_{i}$, for which

$$
L(c)+\lambda(c)=0, \quad L^{\prime}(c)+\lambda^{\prime}(c) \geqq 0 .
$$

As $L^{\prime}(x)<0$ for all values of $x$, we may rewrite these conditions in the form.

$$
\lambda(c)=-L(c), \quad\left|\frac{\lambda(c)}{\lambda^{\prime}(c)}\right| \leqq\left|\frac{L(c)}{L^{\prime}(c)}\right|, \quad \lambda^{\prime}(c)>0,
$$

from which it follows that there must be a point of intersection of the two curves $y=\lambda(x), y=-L(x)$, at which the former is rising, and where its subtangent is less than or at most equal to the subtangent of the latter in absolute value.

The subtangent to $\lambda$ at any point is

$$
\frac{\lambda}{\lambda^{\prime}} \equiv \frac{2 \sum_{i=1}^{k} \frac{x-a_{i}}{\left(x-a_{i}\right)^{2}+b_{i}^{2}}}{2 \sum_{i=1}^{k} \frac{b_{i}^{2}-\left(x-a_{i}\right)^{2}}{\left[b_{i}^{2}+\left(x-a_{i}\right)^{2}\right]^{2}}} .
$$

As we require that every $a$ is to be without and on the same side of the interval, the terms of the numerator are all of the same sign for $x=c$, and, denoting by $\alpha$ the $a$ nearest to $c$, we may write

$$
\begin{aligned}
& |\lambda(c)| \geqq 2 \sum_{i=1}^{k} \frac{|c-\alpha|}{\left(c-a_{i}\right)^{2}+b_{i}^{2}}=2|c-\alpha| \sum_{i=1}^{k} \frac{1}{\left(c-a_{i}\right)^{2}+b_{i}^{2}}, \\
& \left|\lambda^{\prime}(c)\right|<2 \sum_{i=1}^{k} \frac{b_{i}^{2}+\left(c-a_{i}\right)^{2}}{\left[b_{i}^{2}+\left(c-a_{i}\right)^{2}\right]^{2}}=2 \sum_{i=1}^{k} \frac{1}{\left(c-a_{i}\right)^{2}+b_{i}^{2}}, \\
& \left|\frac{\lambda(c)}{\lambda^{\prime}(c)}\right|>|c-\alpha| \text {. }
\end{aligned}
$$

* The opposite end-point of the interval, $r_{h}$ or $r_{i}$, is included in this enumeration. 
Thus the subtangent to $\lambda$ at the point $c$ is greater in absolute value than the distance between $c$ and the nearest real part of an imaginary root of $f$, and as all of these are to lie without the interval while $c$ lies within it, we must have

$\frac{-\lambda(c)}{\lambda^{\prime}(c)}>\alpha-c>r_{i}-c$, if $c<\alpha \leqq a_{1}, a_{2}, \cdots, a_{k}$, so that $\lambda(c)<0$, $\frac{\lambda(c)}{\lambda^{\prime}(c)}>c-\alpha>c-r_{h}$, if $c>\alpha \geqq a_{1}, a_{2}, \cdots, a_{k}$, so that $\lambda(c)>0$.

But by (21) this requires that we should also have

$$
\begin{aligned}
& \frac{-L(c)}{L^{\prime}(c)}>r_{i}-c, \text { if } L(c)>0, \\
& \frac{L(c)}{L^{\prime}(c)}>c-r_{h}, \quad \text { if } \quad L(c)<0 .
\end{aligned}
$$

We proceed, therefore, to determine the conditions under which these latter inequalities can subsist; i. e., in which the tangent drawn to $y=L(x)$ at an interior point of the interval can intersect the $X$-axis outside of that interval.

The intercept on the $X$-axis of the tangent to $y=L(x)$,

$$
\begin{aligned}
& X \equiv x-\frac{L}{L^{\prime}} \\
& \equiv x+\frac{\frac{1}{x-r_{1}}+\cdots+\frac{1}{x-r_{h}}+\frac{1}{x-r_{i}}+\cdots+\frac{1}{x-r_{m}}}{\frac{1}{\left(x-r_{1}\right)^{2}}+\cdots+\frac{1}{\left(x-r_{h}\right)^{2}}+\frac{1}{\left(x-r_{i}\right)^{2}}+\cdots+\frac{1}{\left(x-r_{m}\right)^{2}}},
\end{aligned}
$$

may, for a fixed value of $x$, be regarded as a function of the roots, $r_{1}, \cdots, r_{m}$. Taking the partial derivative of $X$ with respect to any one of them, we have

$$
L^{\prime 2} \cdot \frac{\partial X}{\partial r}=-\frac{L^{\prime}}{(x-r)^{2}}-\frac{2 L}{(x-r)^{3}} .
$$

As $L^{\prime}<0$ for all values of $x$ and $r$, the first term of the second member is always positive. When $L>0$ and $r>x$, or when $L<0$ and $r<x$, the second term is also positive and $(\partial X / \partial r)>0$. Therefore for any point where $L$ is positive, $X$ will be increased by removing indefinitely to the right any real root of $f$ that lies to the right of that point; and for any value of $x$ for which $L$ is negative, $X$ will be made less if we remove indefinitely to the left any real root that lies to the left of $x$. But to remove a root indefinitely to the right or left is ultimately to cause the corresponding term in $L$ and $L^{\prime}$ to vanish. Therefore we obtain an upper and a lower limit for the intercept 
of the tangent drawn to $y=L(x)$ at any point of the interval by disregarding, for the upper limit, all the real roots greater than $r_{i}$, and, for the lower limit, all the real roots less than $r_{h}$.

But when $L>0$ and $r<x$, or when $L<0$ and $r>x$, the second member is composed of two terms of opposite sign, and becomes zero when

$$
r=x+\frac{2 L}{L^{\prime}},
$$

which gives us the maximum value of $X$ in the first case, and the minimum in the second. Substituting this value for each $r$ that is less than $x$ in the expression (25) for $X$, and removing indefinitely to the right all the real roots greater than $r_{i}$, we have, as the greatest value which $X$ can assume when $L$ is positive,

$$
X=x-\frac{L}{L^{\prime}}=x+\frac{-h \frac{L^{\prime}}{2 L}+\frac{1}{x-r_{i}}}{h \frac{L^{\prime 2}}{4 L^{2}}+\frac{1}{\left(x-r_{i}\right)^{2}}},
$$

where $h$ denotes the number of real roots of $f$ that lie to the left of $x$, including the root $r_{h}$ at the beginning of the interval. This gives us,

or, for $x=c$,

$$
\begin{gathered}
-\frac{L}{L^{\prime}}\left(h \frac{L^{\prime 2}}{4 L^{2}}+\frac{1}{\left(x-r_{i}\right)^{2}}\right)=-h \frac{L^{\prime}}{2 L}+\frac{1}{x-r_{i}}, \\
\frac{h}{4} \frac{L^{\prime}}{L}-\frac{1}{x-r_{i}}-\frac{L}{L^{\prime}} \frac{1}{\left(x-r_{i}\right)^{2}}=0,
\end{gathered}
$$

$$
\frac{h}{4}=\frac{1}{r_{i}-c}\left(-\frac{L(c)}{L^{\prime}(c)}\right)+\frac{1}{\left(r_{i}-c\right)^{2}}\left(\frac{L(c)}{L^{\prime}(c)}\right)^{2} .
$$

If now, as (23) requires, we are to have

$$
-\frac{L(c)}{L^{\prime}(c)}>r_{i}-c,
$$

each of the terms of the second member of (27) must be greater than unity, and therefore

$$
\frac{1}{4} h>2, \quad h>8,
$$

and the degree of $\phi$ must be at least 10 .

A precisely similar substitution shows that $X$ cannot fall to the left of $r_{h}$ unless the number of real roots to the right of the interval is greater than 8 , and so, as before, the total number of real roots is at least 10 . 
That the number of imaginary roots of $f$ must be greater than 8 follows from Theorem IV, and the proof is complete.

8. Applications and Examples. To illustrate the application of these theorems we may consider the following simple examples.

Example 1. A graph of the polynomial

$$
3 x^{4}-28 x^{3}+72 x^{2}-110
$$

shows bend points at $x=3$ and $x=4$, whereas its greatest real root lies between 1.9 and 2. By Theorem III, we then have for its imaginary roots,

$$
4<a<5.1, \quad 0<b<1.1 \text {. }
$$

Example 2. The equation

$$
x^{5}-5 x^{4}+10 x^{3}-20 x^{2}+40 x-26=0
$$

has unity for its sole real root, as its derivative vanishes for $x=2,2$, and $\pm i \sqrt{2}$. Of the four imaginary roots, one pair must have its real part negative; and for the other pair we may write by Theorems I and II,

$$
2<a<2.71, \quad 0<b<1 .
$$

Example 3. The equation

$$
48 x^{5}-85 x^{3}+15 x+22=0
$$

has unity for a double root, its remaining real root lying between -1.3 and - 1.4. Its derivative vanishes for $x= \pm 1$ and $\pm \frac{1}{4}$. By the corollary to Theorem IV, we have for the imaginary roots

$$
-1.4<a<1, \quad 0<b<1.2 \text {. }
$$

When the roots of the polynomial itself are known, these theorems may often be useful in determining the nature of the roots of the derivative.

Example 4. The equation

$$
f(x) \equiv\left(x+\frac{7}{8}\right)\left(x-\frac{7}{8}\right)\left[(x+1)^{2}+3\right]^{2} \cdot\left[(x-1)^{2}+3\right]^{2}=0
$$

has the real part of its imaginary roots outside of the interval between its least and greatest real roots. As the number of imaginary roots is not greater than eight, by Theorem IV the derivative can vanish only once within that interval. It cannot vanish on the boundary, as the real roots are not multiple. It cannot vanish outside the interval, for if $\rho>\frac{7}{8}$ were a real root of $f^{\prime}$, by Theorem I we should have $\rho<1$ and $b \leqq \frac{1}{4}$, whereas, on the contrary, $b=\sqrt{3}$. Therefore the derivative can have but one real root; and by symmetry it is zero. 
Example 5. The equation

$$
f(x) \equiv\left(x+\frac{7}{8}\right)\left(x-\frac{7}{8}\right)\left[(x+1)^{2}+3\right]^{6} \cdot\left[(x-1)^{2}+3\right]^{6}=0
$$

differs from that of Example 4 only in the multiplicity of its imaginary roots, but its derivative vanishes at least once between $-\frac{7}{8}$ and 0 , and at least once between 0 and $+\frac{7}{8}$, as well as at the origin.

Example 6. The equation

$$
x(x-1)^{27}\left[\left(x+\frac{1}{16}\right)^{2}+\frac{27}{2} \frac{5}{6}\right]^{60}=0
$$

has all its imaginary roots without and on the same side of the interval between its real roots; but because of the high multiplicity both of the imaginary roots and of the real root on the opposite side of the interval, the derivative vanishes at least once between 0 and $\frac{1}{4}$, and at least once between $\frac{1}{4}$ and 1 , as well as at $x=\frac{1}{4}$. 